\title{
Andreas Nüchter (2009): 3D Robotic Mapping: The Simultaneous Localization and Mapping Problem with Six Degrees of Freedom (Springer Tracts in Advanced Robotics 52)
}

\author{
Springer, Berlin/Heidelberg, 204 pp., ISBN 978-3-540-89883-2, \\ e-ISBN 978-3-540-89884-9
}

\section{Radu Bogdan Rusu}

Published online: 9 June 2010

(C) Springer-Verlag 2010

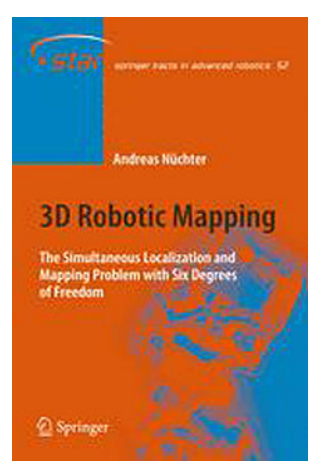

The book 3D Robotic Mapping by Andreas Nüchter represents an excellent reference for any roboticist working in the area of Simultaneous Localization and Mapping, especially those interested in 3D map building.

Throughout its 8 chapters, the reader is first introduced with the basic concepts of $3 \mathrm{D}$ data perception and sensor devices, followed by more advanced topics such as $3 \mathrm{D}$ range image registration and global loop closure for SLAM systems. Additionally, several higher level semantic interpretation methods are presented and evaluated for the purpose of scene and object localization. The theoretical methods presented in the book are solid and anchored in multiple real world experiments.

The book's intended target audience is large, ranging from undergraduate students to postdoctoral researchers keen on learning or working on this topic. By providing the underlying mathematical explanations and proofs for the 3D geometric problems tackled, the reader is most of the time given the complete information required to understand and even implement the presented concepts. The added advantage of this compact formulation is that one doesn't need much additional material (such as extra books, journal papers, etc.).

Perhaps a critic towards the overall organization of the book, is that it sometimes plunges too much into tiny details, while at the same time omitting to explain the bigger overall goal. The experimental section is missing some a few references and results obtained in indoor environments, and is sometimes concentrating too much on outdoor maps. This is natural, as it constitutes a major part of the work that the author has been involved with, but it would be nice to know if some of this concepts can be applied to other application scenarios such as humanoid robots, robots performing manipulation in dynamic environments, and so on. A definitive plus would be to also present disadvantages and failures of the methods presented, rather than expressing them as ideal solutions for a given problem.

Future work should also include a bit more about the overall picture, improvements that the entire community should pull for to solve mapping problems better, faster, and in general more efficient than previous research iterations.

Despite all this, I strongly recommend this book as a good introduction and reference material for 3D perception and mapping with mobile robots. If everyone would know and understand the concepts presented in it, then the community as a whole would have a stronger base from where it could look out for solutions to the more complicated problems that still remain unsolved in this domain. 